
\title{
Effects of reconstruction of the anterior
cruciate ligament on voluntary activation of
quadriceps femoris
}

(a)

A PROSPECTIVE TWITCH INTERPOLATION STUDY

D. Urbach, W. Nebelung, R. Becker, F. Awiszus

From Otto-von-Guericke University, Magdeburg, Germany

$\mathbf{T}$ he loss of full muscle activation contributes to weakness of the quadriceps muscle in patients with deficiency of the anterior cruciate ligament (ACL). We examined whether a deficit of voluntary activation (VA) of the quadriceps muscle can be reversed by reconstruction of the ACL and assessed its influence on muscle strength and clinical parameters.

We evaluated 12 male subjects with an isolated tear of the ACL and 12 matched control subjects before operation and two years after reconstruction of the ACL. Assessment included measurements of isometric knee-extension torque at maximal voluntary contraction (MVC force), knee stability tests, the International Knee Ligament Standard Evaluation Form and the Tegner activity score. A sensitive method of twitch interpolation was used to quantify the VA and to calculate true muscle force.

Before operation we found a deficit of VA on both the injured (mean \pm SEM $74.9 \pm 3.5 \%$ ) and the uninjured side $(74.6 \pm 3.0 \%)$ in comparison with the control group $(91 \pm 0.9 \%)$. Two years after reconstruction of the $A C L$ the $V A$ improved significantly on both sides but remained less than that of the controls. Correlation analysis revealed an improvement of the VA in patients who returned to a higher level of activity. The deficit of true muscle force, however, persisted regardless of the clinical outcome and ligament stability.

J Bone Joint Surg [Br] 2001;83-B:1104-1110. Received 15 August 2000; Accepted after revision 18 April 2001

Rupture of the anterior cruciate ligament (ACL) is one of the more common sports injuries of the knee. ${ }^{1-3}$ Weakness

D. Urbach, MD

W. Nebelung, MD

R. Becker, MD

F. Awiszus, MD, MSc

Neuromuscular Research Group, Department of Orthopaedics, Otto-vonGuericke University, Leipziger Strasse 44, D-39120 Magdeburg, Germany.

Correspondence should be sent to Dr D. Urbach.

(C)2001 British Editorial Society of Bone and Joint Surgery 0301-620X/01/811618\$2.00 of the thigh muscle and wasting are usual in chronic ACL deficiency $^{4,5}$ and after reconstruction. ${ }^{6-9}$ Several studies ${ }^{10,11}$ have demonstrated that muscular rehabilitation to normal strength is difficult, protracted, and often not achieved. It has been shown that the inability fully to activate quadriceps femoris voluntarily contributes to the deficit and may undermine effective physiotherapy. ${ }^{11-15}$ This voluntary activation, VA, is also termed arthrogenous muscle inhibition or reflex inhibition. Furthermore, there is evidence that physiotherapy has no effect on the loss of VA in patients after isolated rupture of the ACL without reconstruction. ${ }^{11,12}$ There has been no prospective study which has determined whether patients benefit from reconstruction of the ACL by regaining their ability to activate their quadriceps muscles through the normal range. New, highlysensitive methods of twitch interpolation detect even subtle deficits of VA. ${ }^{16-18}$ Applying these techniques, it has been shown that most healthy subjects are not able voluntarily to elicit full muscle strength. ${ }^{14,19,20}$ The method of twitch interpolation requires study of a healthy control group in order to determine quantitatively whether the subjects tested already have a deficiency. Our aim therefore was to measure the extent of deficits of VA and of isometric quadriceps force before and after reconstruction of the ACL on the injured and uninjured sides using an established highly-sensitive method of twitch interpolation. ${ }^{16,17,21,22}$ The reliability of measurements of muscle strength and VA using this technique has already been studied. ${ }^{21,23}$ In addition, clinical evaluation was performed to determine the influence of a possible VA deficit on the clinical outcome and to identify clinical parameters which may influence the extent of a deficit and of muscle weakness.

\section{Patients and Methods}

All experimental procedures were approved by the local Ethics Committee and all subjects gave informed consent.

We studied 12 males with isolated rupture of the ACL, with a mean age of 26.9 years (14.9 to 43.5), and 12 matched normal control subjects, with a mean age of 26.4 years (15.3 to 42.3$)$.

The mean time between injury and surgery was 13 months (3 to 21). In every case, the ruptured ACL was reconstructed using quadruple strands of semitendinosus 
tendon by an arthroscopically-assisted technique. The rehabilitation regime after operation was identical for all patients. They were allowed to bear as much weight as they could tolerate, wearing a knee brace which limited knee flexion to between $10^{\circ}$ and $90^{\circ}$ for the first six weeks. Passive continuous movement was performed from $0^{\circ}$ to $90^{\circ}$ of flexion from the third postoperative day. At five months non-contact sport was allowed and contact sport was permitted from the seventh month after operation.

The matching requirements for the control group were gender (all males), age $( \pm 10 \%)$, activity level ( \pm one level of the preinjury Tegner activity score) and a maximal contraction force of the quadriceps muscle equivalent to that of the uninjured side before operation, as described below $( \pm 15 \%)$. The first evaluation was performed one day before surgical reconstruction and the second 21 to 25 months after surgery. Measurements included the ability to activate quadriceps femoris voluntarily, the isometric voluntary contraction force, knee stability using the KT 1000 arthrometer (Medmetrics Inc, San Diego, California), a clinical assessment according to the International Knee Documentation Committee (IKDC) form ${ }^{24}$ and the activity level by the Tegner score. ${ }^{25}$

Twitch-interpolation technique. Patients were seated in an upright position on a purpose-built chair. The lower legs were fixed to the lever arm of a force-measuring device based on an analogue strain gauge. The force signal was amplified, transformed from analogue to digital with a sampling frequency of $1 \mathrm{kHz}$, and converted to kneeextension torque. For electrical stimulation of the muscle aluminium-plate electrodes were strapped to the quadriceps and a constant current was applied (Dantec Counterpoint K II, Skovlunde, Denmark), with single, square-wave stimuli of amplitude $100 \mathrm{~mA}$ and duration $500 \mu \mathrm{s}$. Custom-written software was used for triggering the pulses and for online display of the exerted torque.

The subjects were instructed to extend their knee fully for five seconds to determine the force at maximum voluntary contraction (MVC) measured as extension torque and for maximal potentiation of the twitch response. ${ }^{21}$ Immediately after twitch potentiation, the subjects performed isometric contractions with $90 \%, 75 \%, 50 \%$ and $100 \%$ of their MVC force by matching the visualised torque level on the monitor with the desired torque. When the torque was stable three single stimuli were applied to the muscle.

Providing over $25 \%$ of the MVC force, the additional force generated by the superimposing electrical stimulation is known to have a linear relationship with the voluntary force (Fig. 1). ${ }^{21,22}$ This linearity is used to extrapolate the twitch amplitude from zero MVC ('resting' muscle). The VA of the muscle is calculated using the formula: ${ }^{11,26}$

VA $(\%)=1-\frac{\text { Twitch amplitude at } \operatorname{MVC}(\mathrm{Nm})}{\begin{array}{c}\text { Estimated twitch amplitude at } \\ \text { resting muscle }(\mathrm{Nm})\end{array}} \times 100$
Knowing the MVC force and the VA, the extension torque was calculated for imaginary $100 \%$ muscle activation, representing the 'true' maximum contraction force (TMC force) of quadriceps. Data acquisition and highlysensitive twitch detection were performed uniformly by specific software to exclude observer- and examinerdependent variations using the technique described by Hales and Gandevia. ${ }^{16}$

Statistical analysis. We performed two-tailed non-parametric paired sample tests (Wilcoxon signed-rank) to compare pre- and postoperative data and to compare the injured with the uninjured side. Non-parametric, two independent sample tests (Mann-Whitney U) were used to compare the patients with the control group. To establish correlations between parameters Spearman's rank coefficient of correlation was calculated. The level for statistical significance was set to an alpha level of $\mathrm{p}<0.05$ referring to two-tailed tests. If not specified otherwise, the results are means \pm SEM.

\section{Results}

Clinical evaluation. The evaluation of each knee before operation revealed that in nine patients a grade-D and in three patients a grade-C lesion was present according to the IKDC score. Two years after reconstruction of the ACL there were four grade- $A$, five grade- $B$ and three grade- $C$ knees. Of the three patients with an 'abnormal' knee, one patient described swelling after jogging (IKDC activity level III), one described swelling and pain after jogging and the third complained of partial giving way at activity level III with a subjective assessment of grade $\mathrm{C}$.

Before operation, measurements using the KT 1000 arthrometer revealed mean differences of $4.3 \mathrm{~mm}$ (3 to 7) of anterior displacement at a load of $13.5 \mathrm{~kg}$ between the injured and the uninjured sides in all patients. After surgery this value improved to $1.6 \mathrm{~mm}$ ( -1 to $4 \mathrm{~mm})$. The mean score for the Tegner activity level was 4.2 (1 to 9) before reconstruction and 6.6 (4 to 9) two years after operation; this did not reach the activity level before injury (mean 7.9; range 6 to 9). The mean activity level according to the IKDC scale was 1.18 (1 to 3 ) before injury, 3.45 (1 to 4 ) before surgery, and 1.91 (1 to 3 ) two years after reconstruction of the ACL.

Voluntary activation of the quadriceps femoris. Figure 1 shows a typical result of the twitch-interpolation method in a patient before reconstruction of the ACL (Figs 1a and 1b) compared with the matched control (Figs 1c and 1d). In the patient, a high additional extension torque was elicited at MVC by the electrical twitch. Using the linear relationship between twitch amplitude and initial torque, calculations gave a VA of $72 \%$ on the injured side and $73 \%$ of the uninjured side. By contrast, Figure 1c shows that there was almost no additional extension torque after the electric stimulus at MVC in the matched control subject, giving a VA of $96 \%$ on the uninjured right side and $94 \%$ on the left. 


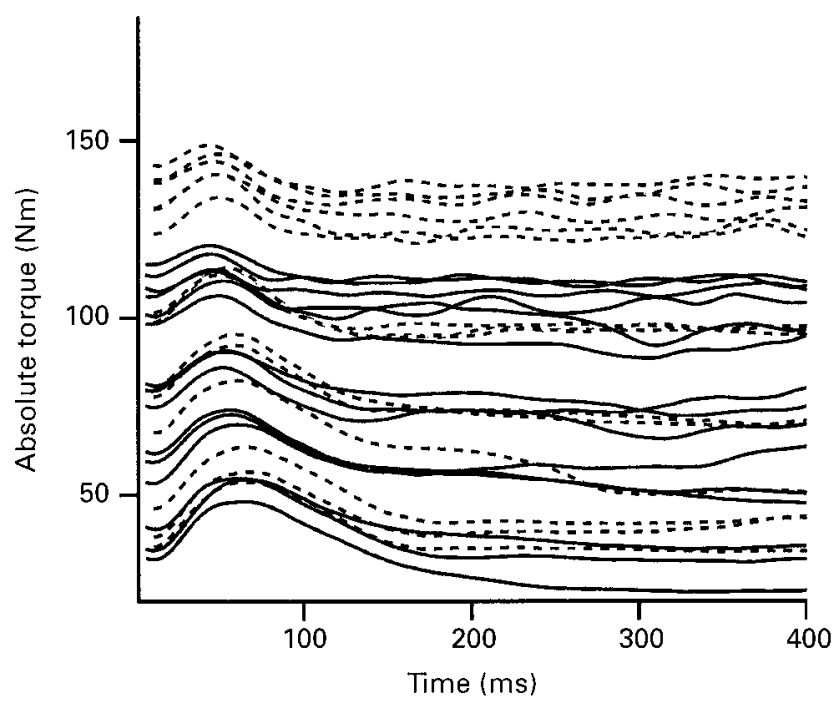

Fig. 1a

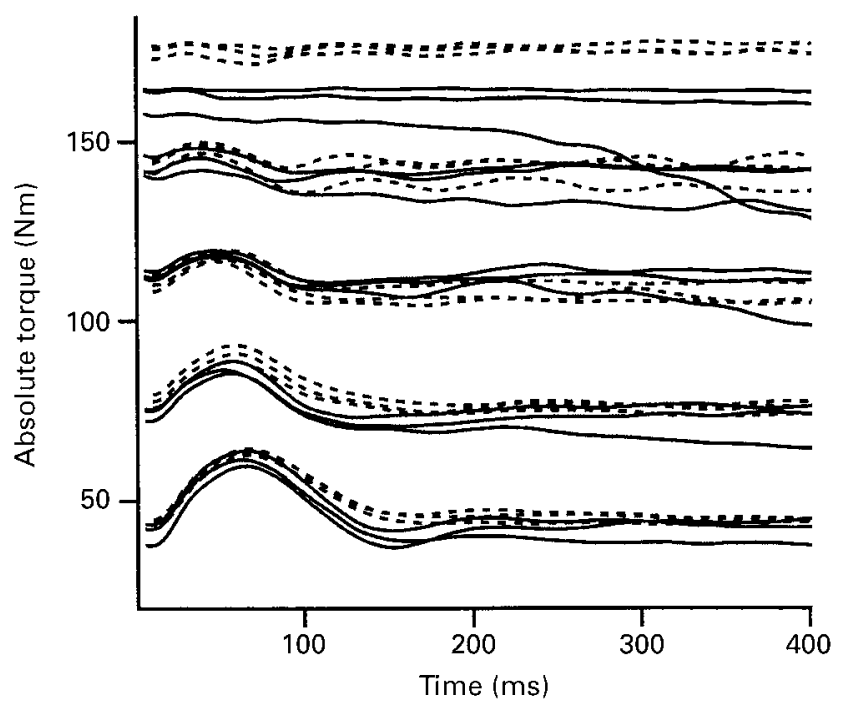

Fig. 1c

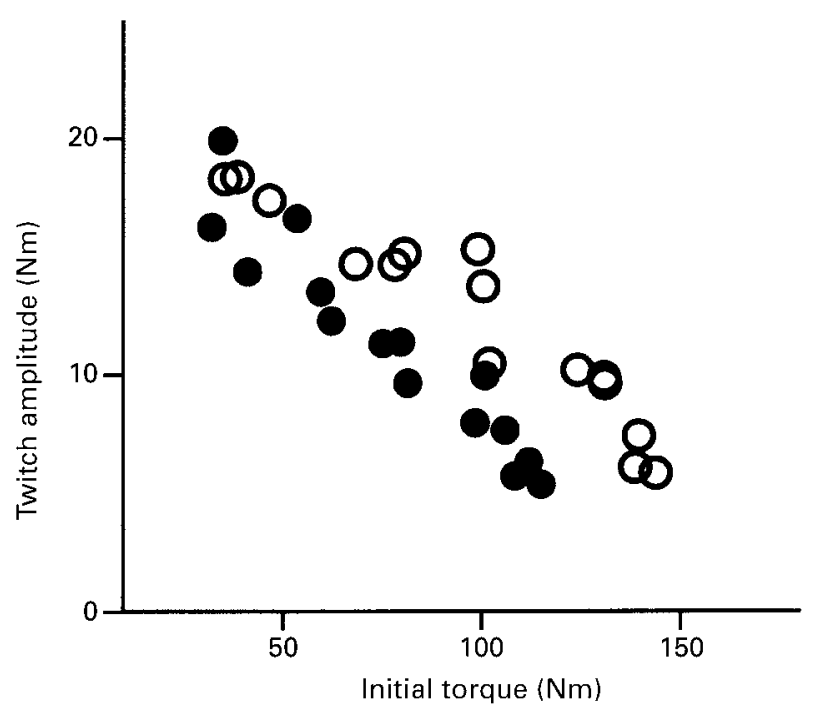

Fig. 1b

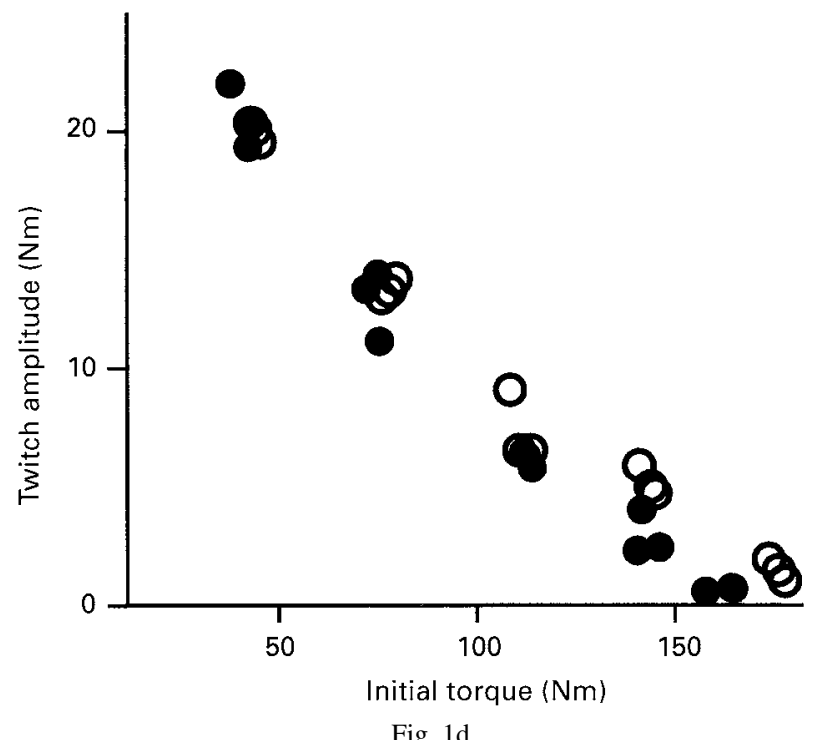

Fig. 1d

Determination of the VA using the twitch interpolation method in a patient before reconstruction of the ACL on the injured side (continuous lines, solid circles) and on the uninjured side (broken lines, open circles (a,b)). For comparison, results are shown for the matched control subject (c,d), who was able to elicit nearly full strength voluntarily. The lines show the knee-extension torque measured for 400 ms after the muscle stimulation at different initial torque values according to the experimental protocol $(\mathrm{a}, \mathrm{c})$. The circles represent the calculated twitch amplitudes at the corresponding initial torque $(b, d)$.

The quadriceps on the injured side of the patient was weaker (MVC force $112 \mathrm{Nm}$ ) than that on the contralateral (right) side $(144 \mathrm{Nm})$ and also than that on the right side of the control subject $(165 \mathrm{Nm})$. The latter met the matching condition of equal TMC force of the quadriceps of the patients on the uninjured side before operation (patient's contralateral TMC force $197 \mathrm{Nm}$; control TMC force $185 \mathrm{Nm}$ ).

The cumulative distribution function in Figure 2 shows a clear leftward shift towards lower VA values on the injured side $(74.9 \pm 3.5 \%, \mathrm{p}<0.001)$ and also on the contralateral side $(74.6 \pm 3.0 \%, \mathrm{p}<0.001)$ before operation (broken line), compared with the control group (dotted lines: corre- sponding to the injured side $90.3 \pm 1.4 \%$, corresponding to the uninjured side $91.0 \pm 1.3 \%$ ). Two years after operation (continuous lines) the ability to activate the quadriceps muscle voluntarily improved significantly (injured side $85.3 \pm 2.5 \%, p=0.006$; uninjured side $84.0 \pm 2.4 \%$, $\mathrm{p}=0.006$ ) but remained lower than the controls, with borderline significance (injured side $p=0.062$, uninjured side $\mathrm{p}=0.01$ ). Minor differences between sides did not reach statistical significance.

No correlation was found between the VA and the IKDC evaluation, the subgroups of the IKDC evaluation, the KT 1000 stability measurements, the time interval between injury and first measurement, or age before or after opera- 


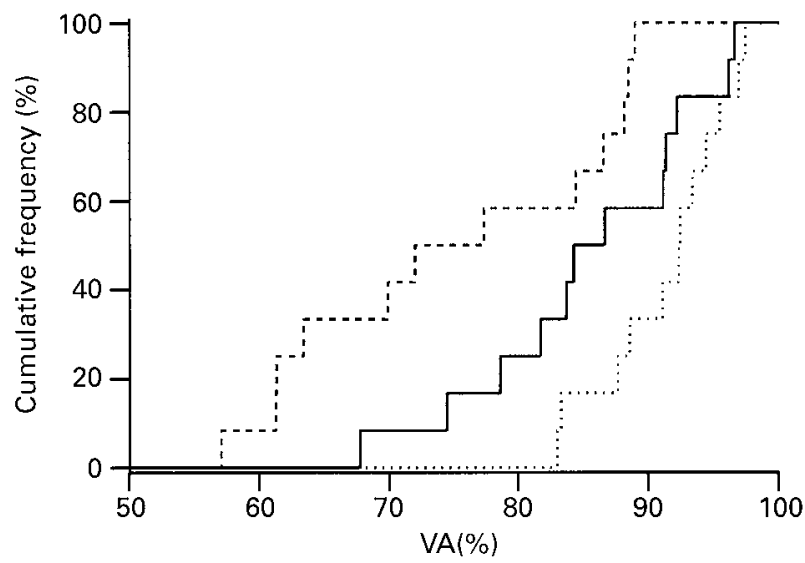

Fig. 2a

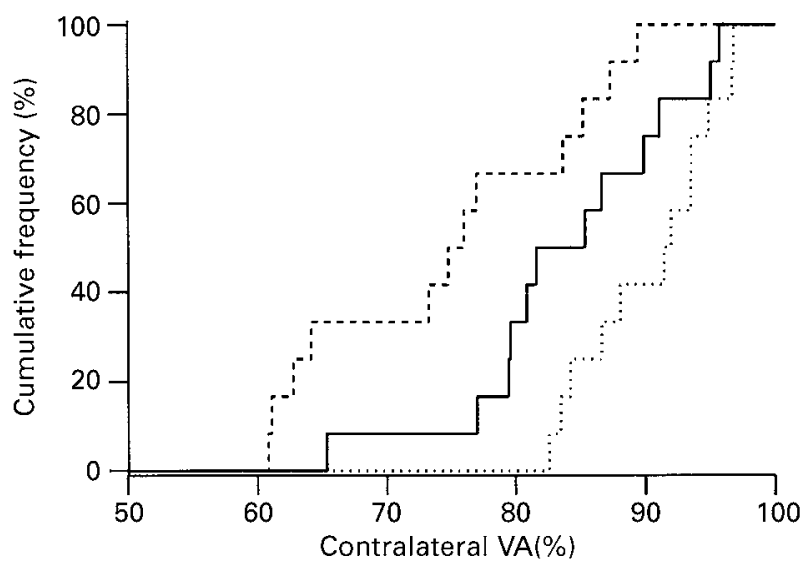

Fig. $2 b$

Cumulative distribution functions of the VA of quadriceps femoris on the injured side (a) and the uninjured side (b) (broken lines, patients before operation; continuous lines patients two years after ACL reconstruction; dotted lines, control subjects).

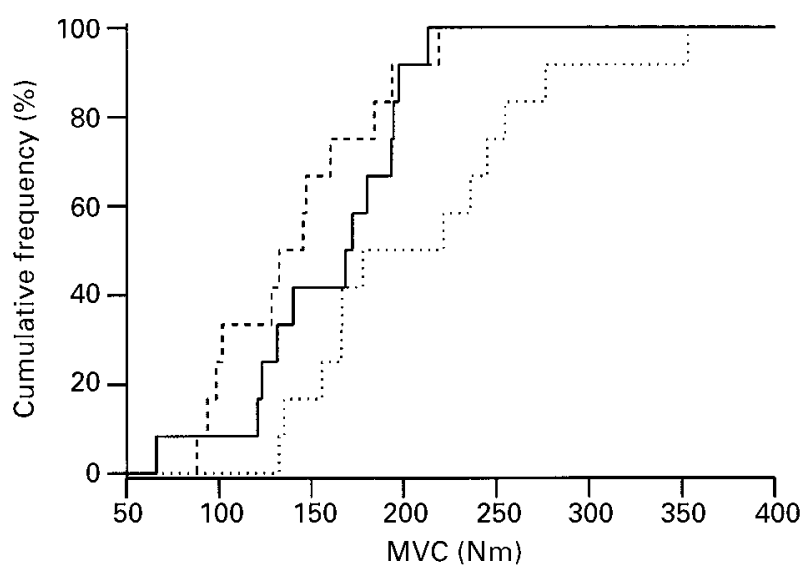

Fig. 3a

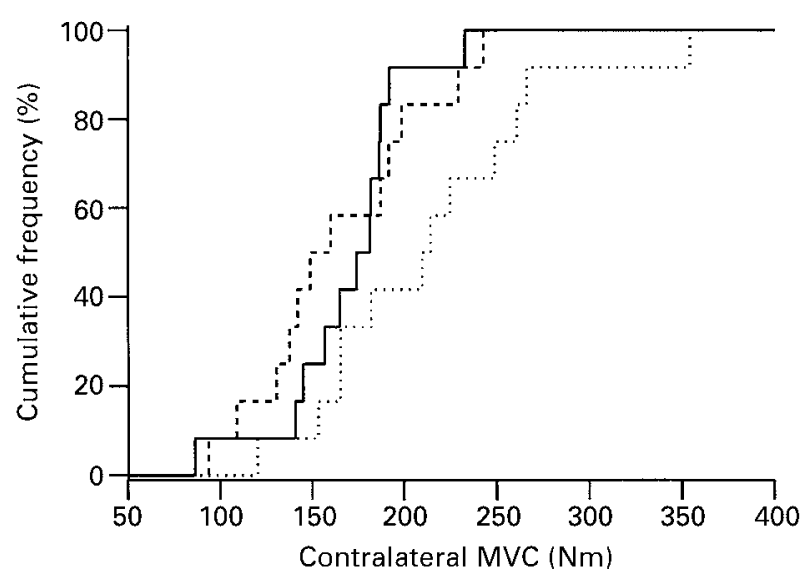

Fig. $3 b$

Cumulative distribution functions of the knee extension torque at MVC of quadriceps femoris on the injured side (a) and the uninjured side (b) (broken lines, patients before operation; continuous lines, patients two years after reconstruction of the ACL; dotted lines control subjects).

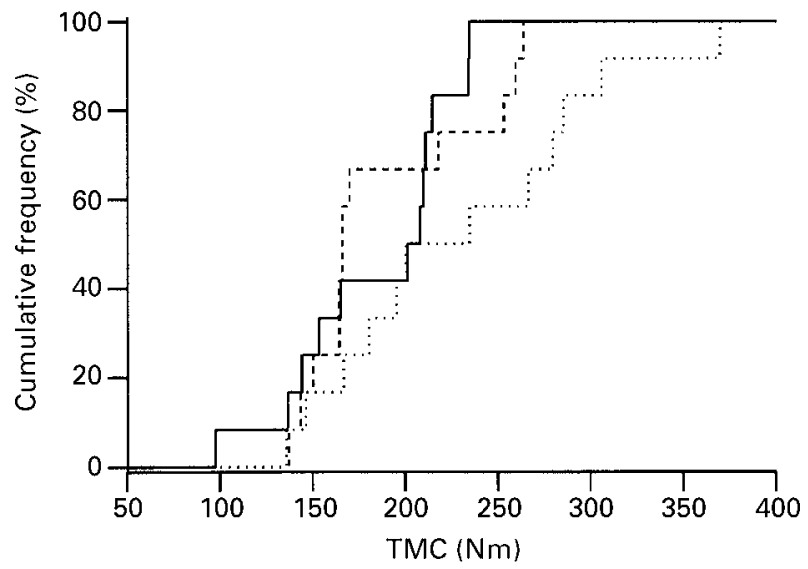

Fig. 4a

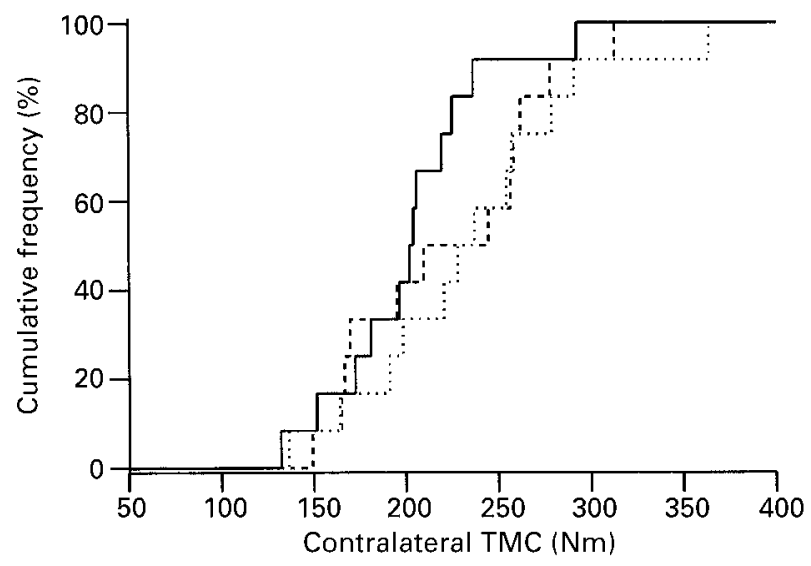

Fig. 4b

Cumulative distribution functions of the calculated TMC force at imaginary full quadriceps muscle activation on the injured side (a) and the uninjured side (b) (broken lines, patients before operation; continuous lines, patients two years after operation; dotted lines, control subjects). 
tion. A significant correlation $(r=0.71, p=0.009)$ was found between the recovery of VA after reconstruction of the ACL and the improvement in the activity level according to the Tegner score.

MVC force and TMC force. The cumulative distribution functions of the MVC forces of the patients before operation (Fig. 3, broken lines) on the injured and uninjured sides show a clear, almost parallel shift towards low MVC forces in comparison with the controls (dotted lines). The lower MVC forces on the injured side $(141.1 \pm 12.2 \mathrm{Nm})$ and on the uninjured side $(164.4 \pm 13.3 \mathrm{Nm})$ differed significantly from those of the corresponding sides in the controls (injured side $214.0 \pm 18.2 \mathrm{Nm}, \mathrm{p}=0.001$; uninjured side $210.0 \pm 19.1 \mathrm{Nm}, \mathrm{p}=0.029)$. The differences between sides were significant $(\mathrm{p}=0.015)$.

Figure 4 shows that the TMC forces of the patients before operation were also significantly lower on the injured side $(188.0 \pm 13.5 \mathrm{Nm}, \mathrm{p}=0.045)$ in comparison with the controls $(235.2 \pm 17.6 \mathrm{Nm})$. The TMC forces in the patients on the uninjured side $(222.4 \pm 15.4 \mathrm{Nm})$ were equal to those of the controls $(230.5 \pm 20.6 \mathrm{Nm})$ according to the matching requirements $(p=0.704)$. Two years after reconstruction of the ACL there was some recovery of the ipsilateral muscle force $(158.4 \pm 12.3 \mathrm{Nm}$, Fig. 3), which did not reach statistical significance $(\mathrm{p}=0.071)$. The MVC forces of the uninjured side remained unchanged two years after reconstruction of the ACL $(169 \pm 10.3 \mathrm{Nm}$, $\mathrm{p}=0.308)$. The TMC forces did not change after surgery on the injured side $(184.2 \pm 12.5 \mathrm{Nm}, \mathrm{p}=0.695)$ and were reduced on the uninjured side after the operation with borderline statistical significance $(201.6 \pm 11.9 \mathrm{Nm}$, $\mathrm{p}=0.05)$.

\section{Discussion}

We studied a group of male patients with instability of the knee after rupture of the ACL. The rehabilitation regime before reconstruction of the ACL was not standardised because the patients were transferred from different centres. After operation a standardised progressive rehabilitation regime was used. All patients benefited from the reconstruction of the ACL, but the high level of sports activity was not entirely restored and three of the 12 knees were rated as 'abnormal' according to the IKDC evaluation. KT 1000 measurements revealed an improvement in stability (side-to-side differences after operation $1.6 \mathrm{~mm}$ ) to the same degree as reported previously using a multistrand semitendinosus tendon graft by Marder, Raskind and Car$\operatorname{roll}^{27}(1.9 \mathrm{~mm})$, Maeda et $\mathrm{al}^{28}(1.5 \mathrm{~mm})$ and Muneta et $\mathrm{al}^{29}$ $(2.3 \mathrm{~mm})$. Comparability is slightly prejudiced by minor variations in surgical technique, modes of evaluation and differing rehabilitation regimes, which could influence the measurements of stability. ${ }^{29}$

A major finding is that before operation the patients presented a clear VA deficit and that this improved significantly after reconstruction of the ACL without, how- ever, reaching the levels of their matched controls. Hurley et $\mathrm{al}^{11}$ investigated the effect of physiotherapy on VA deficits in ten subjects with subacute or chronic, isolated deficiency of the ACL. They found a lesser VA deficit before (mean 91\%) and after physiotherapy (mean 94\%) than in the subjects in our study, who showed a mean VA of $75 \%$ before operation. These differences, however, are to be expected considering the different study population. Their subjects were treated conservatively by physiotherapy. By contrast, the patients in our study had not benefited from physiotherapy, and had clinical indications for reconstruction. Furthermore, Hurley et al ${ }^{11}$ used a different, less sensitive method of twitch interpolation, compared with our technique which was developed by Hales and Gandevia. ${ }^{16}$ Applying the technique of Hurley et al, it would be assumed that all normal subjects are able to activate their muscles fully. However, the control subjects in our study, being well-motivated males without orthopaedic or neurological pathology, achieved a mean VA of only $91 \%$. This observation is supported by the finding reported in recent studies applying highly-sensitive methods of twitch interpolation, that even healthy individuals are commonly not able voluntarily to elicit full muscle strength. ${ }^{19,20,23}$

A semiquantitative technique of twitch interpolation was used by Snyder-Mackler et $\mathrm{al}^{30}$ in a retrospective study to investigate VA deficits in three groups of mixed-gender patients with isolated rupture of the ACL. They found that out of 12 patients with subacute deficiency of the ACL there was a significant VA deficit of the quadriceps muscles, whereas no VA deficit was detected in patients with chronic deficiency of the ACL, nor was any detected after reconstruction. This observation supports our finding that VA deficit improves significantly after reconstruction. The fact that Snyder-Mackler et $\mathrm{al}^{30}$ did not detect a residual VA deficit even in the phase of rehabilitation after reconstruction, may be explained by their less sensitive technique of twitch interpolation.

In our study, the distribution functions of the VA of the patients show that before operation about one-third had a VA within the same range as that of the controls, while after operation this was the case for about two-thirds. We attempted to find indicators for severe preoperative VA deficiency and for a good or poor postoperative improvement in VA. The only link found, however, was between the VA and the level of activity of the patient. Those patients who regained a greater VA performance after reconstruction had a better chance of returning to their preinjury level of activity compared with those showing less of an improvement in VA. It may be argued that we do not know whether the activity level was influenced by the deficit or whether those patients who returned to higher activity levels used their quadriceps femoris muscles vigorously, producing a training effect. The finding of Hurley et $\mathrm{al}^{11,12}$ that even intense physiotherapy has no influence on VA deficit, suggests a direct influence of VA deficiency on 
sporting activity. However, neither our study nor that of Hurley et al was adequately structured to assess the effectiveness of a particular rehabilitation regime. It remains unclear therefore whether specific modalities like isokinetic muscle training, electrical stimulation, transcranial magnetic stimulation ${ }^{31}$ or proprioceptive training have an effect on VA deficiency.

Patients performed slightly better when the knee was more stable, according to KT 1000 measurements. The three knees which were graded ' $A$ ' had side-to-side differences of less than $1 \mathrm{~mm}$, but the correlation was not significant. The patient with the worst stability $(4 \mathrm{~mm}$ difference) returned to preinjury sporting activity (soccer) despite the fact that he complained of occasional givingway of the knee. A similar finding was published recently by Eastlack, Axe and Snyder-Mackler, ${ }^{32}$ who found no correlation between measurable stability and functional outcome after rupture of the ACL. In accordance with previous investigations, the pre- and postoperative VA performance was not related to pain. ${ }^{33,34}$

Before reconstruction we observed a clear deficit of the MVC and the TMC force, on the injured side compared with the contralateral knee and with the controls. The moderate improvement of the MVC force after operation is explained by the recovery of the VA, while the pure muscle performance, characterised by the TMC force, remained unchanged. The finding of persistent muscle weakness, regardless of good clinical parameters, is in accordance with other recent studies. ${ }^{28,32,35}$

Recent studies also provide evidence that reconstruction of the ACL after isolated rupture does not necessarily restore the knee to its preinjury level of physiological function, nor prevent degenerative changes, even if the normal stability of the knee has been restored. ${ }^{3,35,36}$ Dye $^{37}$ developed the concept of the 'envelope of function' which incorporates and connects the principle of load transference and tissue homeostasis in order to represent visually the functional capacity of the knee. The envelope is defined as the load and frequency distribution within a safe range to ensure overall tissue homeostasis. In this context, a VA deficit after a joint injury may be a central regulatory attempt by the knee to inhibit muscle groups moving an injured joint in order to ensure that loading under the critical 'envelope' will not lead to further joint or softtissue damage. The theory of a central nervous inhibition after joint damage is supported by the finding of bilateral VA deficits, and by experimental evidence for a tonic descending inhibition through spinal neurones induced by artificial arthritis of the knee. ${ }^{38,39}$ Another recent study ${ }^{40}$ has shown that proprioceptive deficiency after rupture of the ACL correlates with a loss of cortical somatosensory evoked potentials, emphasising the important role of neurophysiological, cortical mechanisms on musculoskeletal function after ligamentous damage.

It is still unclear why some patients suffer from VA deficiency after rupture of the ACL while others do not. It is also unclear whether the modest VA deficit, two years after successful restoration of knee stability, is responsible for the persistent true muscle weakness of quadriceps femoris on the injured side. Further research is required to investigate the underlying neurophysiological mechanisms. A better understanding of these mechanisms may enable us not only restore the mechanical defect but also to restore the central motor drive to normal.

This research was supported by Deutsche Forschungsgemeinschaft grant AW/2-1 and AW/2-2.

No benefits in any form have been received or will be received from a commercial party related directly or indirectly to the subject of this article.

\section{$\underline{\text { References }}$}

1. Kannus P, Jarvinen M. Incidence of knee injuries and the need for further care: a one-year prospective follow-up study. J Sports Med Phys Fitness 1989;29:321-5.

2. Swenson TM, Fu FH. Anterior cruciate ligament reconstruction: longterm results using autograft tissue. Clin Sports Med 1993;12:709-22.

3. Dye SF, Wojtys EM, Fu FH, Fithian DC, Gillquist I. Factors contributing to function of the knee joint after injury or reconstruction of the anterior cruciate ligament. Instr Course Lect 1999;48:185-98.

4. Kannus P, Jarvinen M. Knee ligament injuries in adolescents: eight year follow-up of conservative management. J Bone Joint Surg [Br] 1988;70-B:772-6.

5. Lorentzon R, Elmqvist LG, Sjostrom M, Fagerlund M, Fuglmeyer AR. Thigh musculature in relation to chronic anterior cruciate ligament tear: muscle size, morphology and mechanical output before reconstruction. Am J Sports Med 1989;17:423-9.

6. Natri A, Jarvinen M, Latvala K, Kannus P. Isokinetic muscle performance after anterior cruciate ligament surgery: long-term results and outcome predicting factors after primary surgery and late-phase reconstruction. Int J Sports Med 1996;17:223-8.

7. Elmqvist LG, Lorentzon R, Johansson C, Fugl-Meyer AR. Does a torn anterior cruciate ligament lead to change in the central nervous drive of the knee extensors? Eur J Appl Physiol Occup Physiol 1988;58:203-7.

8. Snyder-Mackler L, Binder-Macleod SA, Williams PR. Fatigability of human quadriceps femoris muscle following anterior cruciate ligament reconstruction. Med Sci Sports Exerc 1993;25:783-9.

9. Hiemstra LA, Webber S, MacDonald PB, Kriellaars DJ. Knee strength deficits after hamstring tendon and patellar tendon anterior cruciate ligament reconstruction. Med Sci Sports Exerc 2000;32:1472-9.

10. Kannus P, Jarvinen M. Thigh muscle function after partial tear of the medial ligament compartment of the knee. Med Sci Sports Exerc 1991;23;4-9.

11. Hurley MV, Jones DW, Wilson D, Newham DJ. Rehabilitation of quadriceps inhibited due to isolated rupture of the anterior cruciate ligament. J Orthop Rheum 1992;5:145-54.

12. Hurley MV, Jones DW, Newham DJ. Arthrogenic quadriceps inhibition and rehabilitation of patients with extensive traumatic knee injuries. Clin Sci (Colch.) 1994;86:305-10.

13. Stokes M, Young A. The contribution of reflex inhibition to arthrogenous muscle weakness. Clin Sci 1984;67:7-14.

14. Urbach D, Nebelung W, Weiler HT, Awiszus F. Bilateral deficit of voluntary quadriceps muscle activation after unilateral ACL tear. Med Sci Sports Exerc 1999;31:1691-6.

15. Urbach D, Nebelung W, Ropke M, Becker R, Awiszus F. Bilateral dysfunction of the quadriceps muscle after unilateral cruciate ligament rupture with concomitant injury central activation deficit. Unfallchirurg 2000;103:949-55.

16. Hales JP, Gandevia SC. Assessment of maximal voluntary contraction with twitch interpolation: an instrument to measure twitch responses. J Neurosci Methods 1988;25:97-102.

17. Awiszus F, Wahl B, Meinecke I. Influence of stimulus cross talk on results of the twitch-interpolation technique at the biceps brachii muscle. Muscle Nerve 1997;20:1187-90.

18. Allen GM, Gandevia SC, Neering IR, et al. Muscle performance, voluntary activation and perceived effort in normal subjects and patients with prior poliomyelitis. Brain 1994;117:661-70. 
19. Dowling JJ, Konert E, Ljucovic P, Andrews DM. Are humans able to voluntarily elicit maximum muscle force? Neurosci Lett 1994;179:25-8.

20. Yue GH, Ranganathan VK, Siemionow V, Liu JZ, Sahgal V. Evidence of inability to fully activate human limb muscle. Muscle Nerve 2000;23:376-84.

21. Bulow PM, Norregaard J, Danneskiold-Samsoe B, Mehlsen J. Twitch interpolation technique in testing of maximal muscle strength: influence of potentiation, force level, stimulus intensity and preload. Eur J Appl Physiol Occup Physiol 1993;67:462-6.

22. Norregaard J, Lykkegaard JJ, Bulow PM, Danneskiold-Samsoe B. The twitch interpolation technique for the estimation of true quadriceps muscle strength. Clin Physiol 1997;17:523-32.

23. Allen GM, Gandevia SC, McKenzie DK. Reliability of measurements of muscle strength and voluntary activation using twitch interpolation. Muscle Nerve 1995;18:593-600.

24. Hefti F, Müller W, Jakob RP, Staubli HU. Evaluation of knee ligament injuries with the IKDC form. Knee Surg Sports Traumatol Arthrosc 1993;1:226-34.

25. Tegner Y, Lysholm J. Rating systems in the evaluation of bone ligament injuries. Clin Orthop 1985;43-9.

26. Bigland-Ritchie BR, Dawson NJ, Johansson RS, Lippold OC. Reflex origin for the slowing of motoneurone firing rates in fatigue of human voluntary contractions. J Physiol 1986;379:451-9.

27. Marder RA, Raskind JR, Carroll M. Prospective evaluation of arthroscopically assisted anterior cruciate ligament reconstruction: patellar tendon versus semitendinosus and gracilis tendons. Am J Sports Med 1991;19:478-84.

28. Maeda A, Shino K, Horibe S, Nakata K, Buccafusca G. Anterior cruciate ligament reconstruction with multistranded autogenous semitendinosus tendon. Am J Sports Med 1996;24:504-9.

29. Muneta T, Sekiya I, Ogiuchi T, et al. Effects of aggressive early rehabilitation on the outcome of anterior cruciate ligament reconstruction with multi-strand semitendinosus tendon. Int Orthop 1998;22:352-6.
30. Snyder-Mackler L, De Luca PF, Williams PR, Eastlack ME, Bartolozzi AR 3rd. Reflex inhibition of the quadriceps femoris muscle after injury or reconstruction of the anterior cruciate ligament. J Bone Joint Surg [Am] 1994;76-A:555-60.

31. Urbach D, Awiszus F. Effects of transcranial magnetic stimulation on results of the twitch interpolation technique. Muscle Nerve 2000;23:1125-8.

32. Eastlack ME, Axe J, Snyder-Mackler L. Laxity, instability and functionl outcome after ACL injury: copers versus noncopers. Med Sci Sports Exerc 1999;31:210-5.

33. Shakespeare DT, Stokes M, Sherman KP, Young A. Reflex inhibition of the quadriceps after meniscectomy: lack of association with pain. Clin Physiol 1985;5:137-44.

34. Jones DW, Jones DA, Newham DJ. Chronic knee effusion and aspiration: the effect on quadriceps inhibition. $\mathrm{Br} J$ Rheumatol 1987;26:370-4.

35. Frank CB, Jackson DW. The science of reconstruction of the anterior cruciate ligament. J Bone Joint Surg [Am] 1997;79-A:1556-76.

36. Daniel DM, Stone ML, Dobson BE, et al. Fate of the ACL-injured patient: a prospective outcome study. Am J Sports Med 1994;22:632-44.

37. Dye SF. The knee as a biologic transmission with an envelope of function: a theory. Clin Orthop 1996;325:10-8.

38. Cervero F, Schaible HG, Schmidt RF. Tonic descending inhibition of spinal cord neurones driven by joint afferents in normal cats and in cats with an inflamed knee joint. Exp Brain Res 1991;83:675-8.

39. Schaible HG, Neugebauer V, Cervero F, Schmidt RF. Changes in tonic descending inhibition of spinal neurons with articular input during the development of acute arthritis in the cat. $J$ Neurophysiol 1991;66:1021-32.

40. Vleriani M, Restuccia D, DiLazzaro V, et al. Central nervous system modifications in patients with lesion of the anterior cruciate ligament of the knee. Brain 1996;119:1751-62. 